\title{
Spectroscopy Results from FOCUS
}

\author{
Stefano Bianco* on behalf of the FOCUS and E687 Collaboration ${ }^{\dagger}$ \\ Laboratori Nazionali di Frascati dell'INFN - via E. Fermi 40, Frascati I-00044 \\ E-mail: 'stefano.bianco@inf.infn. it',
}

ABSTRACT: We report the preliminary measurement by the FOCUS Collaboration (E831 and E687 at Fermilab) of masses and widths of $\mathrm{L}=1$ charm mesons, including the first observation of the decay mode $D_{s 2}^{*} \rightarrow D^{+} K_{S}^{0}$. We also report a light meson result from the E687 dataset, i.e., the observation of a narrow dip structure in diffractive photoproduction of the $3 \pi^{+} 3 \pi^{-}$final state.

In this paper we present preliminary results from the FOCUS experiment (E831 at Fermilab) on the spectroscopy of bound states of a charm quark and a lighter quark with orbital angular momentum $L=1$. We also report the observation of a narrow dip structure at $1.9 \mathrm{GeV} / \mathrm{c}^{2}$ in a study of diffractive photoproduction of the $3 \pi^{+} 3 \pi^{-}$final state performed by FOCUS predecessor, E687 at Fermilab.

\section{Measurement of masses and widths of $\mathrm{L}=1$ charm mesons}

A consistent theoretical framework for the spectrum of heavy-light mesons is given by the ideas of Heavy Quark Symmetry (HQS), later generalized by Heavy Quark Effective Theory

\footnotetext{
${ }^{*}$ Speaker.

${ }^{\dagger}$ Coauthors are: P.L. Frabetti (INFN and Bologna), J.M. Link, V.S. Paolone, M. Reyes, P.M. Yager (UC DAVIS); J.C. Anjos, I. Bediaga, C. Göbel, J. Magnin, J.M. de Miranda, I.M. Pepe, A.C. dos Reis, F. Simão (CPBF, Rio de Janeiro); S. Carrillo, E. Casimiro, H. Mendez, A.Sánchez-Hernández,, C. Uribe, F. Vasquez (CINVESTAV, México City); L. Cinquini, J.P. Cumalat, C. Dallapiccola, J.F. Ginkel, J.E. Ramirez, B. O'Reilly, E.W. Vaandering (CU Boulder); J.N. Butler, H.W.K. Cheung, S. Cihangir, I. Gaines, P.H. Garbincius, L.A. Garren, E. Gottschalk, S.A. Gourlay, D.J. Harding, P.H. Kasper, A.E. Kreymer, R. Kutschke, P. Lebrun, S. Shukla, M. Vittone (Fermilab); R. Baldini-Ferroli, S. Bianco, F.L. Fabbri, S. Sarwar, A. Zallo (INFN Frascati); C. Cawlfield, R. Culbertson, R. Greene, D.Y. Kim, K.S. Park, A. Rahimi, J. Wiss (UI Champaign); R. Gardner (Indiana ); Y.S. Chung, J.S. Kang, B.R. Ko, J.W. Kwak, K.B. Lee, S.S. Myung, H. Park (Korea University, Seoul); G. Alimonti, G. Bellini, M. Boschini, D. Brambilla, B. Caccianiga, A. Calandrino, L. Cinquini, P. D'Angelo, M. DiCorato, P. Dini, M. Giammarchi, P. Inzani, F. Leveraro, S. Malvezzi, D. Menasce, E. Meroni, M. Mezzadri, L. Milazzo, L. Moroni, D. Pedrini, L. Perasso, F. Prelz, M. Rovere, A. Sala, S. Sala D. Torretta (INFN and Milano); D. Buchholz, D. Claes, B. Gobbi (Nortwestern); J.M. Bishop, N.M. Cason, C.J. Kennedy, G.N. Kim, T.F. Lin, D.L. Puseljic, R.C. Ruchti, W.D. Shepard, J.A. Swiatek, Z.Y. Wu (Notre Dame); T.F. Davenport III (UNC Asheville); V. Arena, G. Boca, G. Bonomi, C. Castoldi, G. Gianini, G. Liguori, M. Merlo, D. Pantea, S.P. Ratti, C. Riccardi, P. Torre, L. Viola, P. Vitulo (INFN and Pavia); H. Hernandez, A.M. Lopez, L. Mendez, A. Mirles, E. Montiel, D. Olaya, J. Quinones, C. Rivera, Y. Zhang (Mayaguez, Puerto Rico); N. Copty, M. Purohit, J.R. Wilson (USC Columbia); K. Cho, T. Handler (UT Knoxville); D. Engh, W.E. Johns, M. Hosack, M.S. Nehring, M. Sales, P.D. Sheldon, K. Stenson, M.S. Webster (Vanderbilt); M. Sheaff (Wisconsin, Madison); Y. Kwon (Yonsei University, Korea).
} 
in the QCD framework. The basic idea is that in the limit of infinite heavy quark mass: a) the much heavier quark does not contribute to the orbital degrees of freedom, which are completely defined by the light quark(s) only; and b) properties are independent of heavy quark flavor. Heavy Quark Symmetry provides explicit predictions on the spectrum of excited charmed states [i] [1] $]$. In the limit of infinite heavy quark mass, the spin of the heavy quark $\mathbf{S}_{\mathbf{Q}}$ decouples from the light quark degrees of freedom ( $\operatorname{spin} \mathbf{s}_{\mathbf{q}}$ and orbital $\mathbf{L}$ ), with $\mathbf{S}_{\mathbf{Q}}$ and $\mathbf{j}_{\mathbf{q}} \equiv \mathbf{s}_{\mathbf{q}}+\mathbf{L}$ the conserved quantum numbers. Predicted excited states are formed by combining $\mathbf{S}_{\mathbf{Q}}$ and $\mathbf{j}_{\mathbf{q}}$. For $L=1$ we have $j_{q}=1 / 2$ and $j_{q}=3 / 2$ which, combined with $S_{Q}$, provide prediction for two $j_{q}=1 / 2(\mathrm{~J}=0,1)$ states, and two $j_{q}=3 / 2(\mathrm{~J}=1,2)$ states. These four states are named respectively $D_{0}^{*}, D_{1}\left(j_{q}=1 / 2\right), D_{1}\left(j_{q}=3 / 2\right)$ and $D_{2}^{*}$. Finally, parity and angular momentum conservation favor the $\left(j_{q}=1 / 2\right)$ states to decay to the ground states mainly via S-wave transitions (broad width), while $\left(j_{q}=3 / 2\right)$ states would decay via $\mathrm{D}$-wave (narrow width). While the narrow states are well established, the evidence for the broad states (both in the $c$-quark and in the $b$-quark sector) is much less stringent [i2i].

The data for this paper were collected in the Wideband photoproduction experiment FOCUS during the last Fermilab fixed-target run. FOCUS is a considerably upgraded ver-

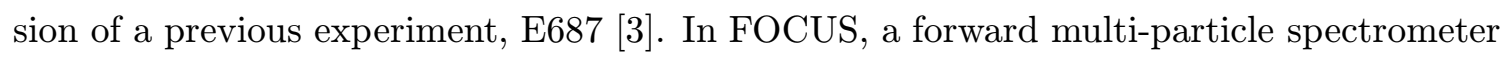
is used to measure the interactions of high energy photons on a segmented $\mathrm{BeO}$ target. We obtained a sample of over 1 million fully reconstructed charm particles in the three major decay modes: $D^{0} \rightarrow K^{-} \pi^{+}, K^{-} \pi^{+} \pi^{-} \pi^{+}$and $D^{+} \rightarrow K^{-} \pi^{+} \pi^{+}$.

The FOCUS detector is a large aperture, fixed-target spectrometer with excellent vertexing, particle identification, and reconstruction capabilities for photons and $\pi^{0}$ 's. A photon beam is derived from the bremsstrahlung of secondary electrons and positrons with an $\approx 300 \mathrm{GeV}$ endpoint energy produced from the $800 \mathrm{GeV} / c$ Tevatron proton beam. The charged particles which emerge from the target are tracked by two systems of silicon microvertex detectors. The upstream system, consisting of 4 planes (two views in 2 stations), is interleaved with the experimental target, while the other system lies downstream of the target and consists of twelve planes of microstrips arranged in three views. These detectors provide high resolution separation of primary (production) and secondary (decay) vertices with an average proper time resolution of $\approx 30 \mathrm{fs}$ for 2 -track vertices. The momentum of a charged particle is determined by measuring its deflections in two analysis magnets of opposite polarity with five stations of multiwire proportional chambers. Three multicell threshold Čerenkov counters are used to discriminate between pions, kaons, and protons.

To measure masses and widths of structures in $D \pi$ final states the decays $D^{0} \rightarrow K^{-} \pi^{+}$, $D^{+} \rightarrow K^{-} \pi^{+} \pi^{+}, D^{0} \rightarrow K^{-} \pi^{+} \pi^{-} \pi^{+}, D^{*+} \rightarrow D^{0} \pi^{+}$were selected. Details of event selection are discussed in $A_{-1}$. The $D^{+}$or $D^{0}$ candidates were combined with the pion tracks in the primary vertex to form $\mathrm{L}=1 D$-meson candidates. Figure $\underline{1}_{1}^{1}$ (left) shows the distribution in the invariant mass difference $\Delta M_{0} \equiv M\left[\left(K^{-} \pi^{+} \pi^{-}\right) \pi^{-}\right]-M\left(K^{-} \pi^{+} \pi^{-}\right)+$ $M_{P D G}\left(D^{+}\right)$. The plot shows a pronounced peak, consistent with being due to a $D_{2}^{* 0}$ of mass $M \approx 2460 \mathrm{MeV} / c^{2}$. Because of the narrow width, this state has traditionally been identified as the $J=2^{+}$state. The additional enhancement at $M \approx 2300 \mathrm{MeV} / c^{2}$ is consistent, as verified from Monte Carlo simulations, with arising from the feed-down 
Table 1: Preliminary measurements of masses and widths for narrow structures in $D^{+} \pi^{-}, D^{0} \pi^{+}$, $D^{0} K^{+}$and $D^{+} K_{S}^{0}$ invariant mass distributions.

\begin{tabular}{|l|l|l|c|c|}
\hline State & \multicolumn{2}{|c}{ Mass $\left(\mathrm{MeV} / \mathrm{c}^{2}\right)$} & \multicolumn{2}{c|}{ Width $\left(\mathrm{MeV} / \mathrm{c}^{2}\right)$} \\
& FOCUS & \multicolumn{1}{c}{ PDG } & FOCUS & PDG \\
\hline$D_{2}^{* 0}$ & $2463.5 \pm 1.5($ stat $) \pm 1.5($ syst $)$ & $2458.9 \pm 2.0$ & $30.5 \pm 1.9($ stat $) \pm 3.8($ syst $)$ & $23 \pm 5$ \\
$D_{2}^{*+}$ & $2468.2 \pm 1.5($ stat $) \pm 1.4($ syst $)$ & $2459 \pm 4$ & $28.6 \pm 1.3($ stat $) \pm 3.8($ syst $)$ & $25_{-7}^{+8}$ \\
$D_{s 2}^{*}$ & $2567.3 \pm 1.3($ stat $)$ & $2573.5 \pm 1.7$ & $28_{-4}^{+5}($ stat $)$ & $15 \pm 5$ \\
$D_{2}^{*+}$ & $2535.1 \pm 0.3($ stat $)$ & $2535.35 \pm 0.34$ & $1.6 \pm 1.0($ stat $)$ & $<2.3 @ 90 \% C L$ \\
\hline
\end{tabular}

of the states $D_{1}^{0}$ and $D_{2}^{* 0}$ decaying to $D^{*+} \pi^{-}$, with the $D^{*+}$ subsequently decaying to $D^{+}$and undetected neutral pion. The $D_{2}^{* 0}$ signal was fitted with a relativistic $D$-wave Breit-Wigner function, convoluted with a gaussian resolution function $(\sigma=7 \mathrm{MeV})$. The background was fitted with the sum of an exponential, and two gaussians for the feeddowns described above, whose peaks and widths were fixed at the Monte Carlo values. The slope of the exponential was fixed to the value determined by a fit to the wrongside events mass distribution, which is very well described by a single-slope exponential in the entire fitting interval $2250-3000 \mathrm{MeV} / c^{2}$. For this fit we get a $\chi^{2} /$ dof $=2$, and a $\Gamma=55 \pm 3 \mathrm{MeV} / c^{2} D_{2}^{* 0}$ width statistically non compatible with the PDG world average of $\Gamma=23 \pm 5 \mathrm{MeV} / c^{2}$. We then add an $S$-wave relativistic Breit-Wigner function to the fit, which improves the fit quality $\chi^{2} /$ dof $=0.9$, and provides a width $\Gamma=30 \pm 2 \mathrm{MeV} / c^{2}$ compatible to the PDG value. The mass difference $\Delta M_{+} \equiv M\left[\left(K^{-} \pi^{+}, K^{-} \pi^{+} \pi^{-} \pi^{+}\right) \pi^{+}\right]-$ $M\left(K^{-} \pi^{+}, K^{-} \pi^{+} \pi^{-} \pi^{+}\right)+M_{P D G}\left(D^{0}\right)$ spectrum (Fig. ${ }_{-1}^{1} ;$ right) shows structures similar to those in the $\Delta M_{0}$ spectrum. The prominent peak is consistent with being due to a $D_{2}^{*+}$ of mass $M \approx 2460 \mathrm{MeV} / c^{2}$. The additional enhancement at $M \approx 2300 \mathrm{MeV} / c^{2}$ is consistent, as verified from Monte Carlo simulations, with arising from the feed-down of the states $D_{1}^{0}$ and $D_{2}^{* 0}$ decaying to $D^{* 0} \pi^{+}$, with the $D^{* 0}$ subsequently decaying to $D^{0}$ and undetected neutral pion. The fitting procedure for the $\Delta M_{+}$spectrum follows the same guidelines as the $\Delta M_{0}$. Several systematics checks have been performed to verify the stability of our measurements of masses and widths, such as fit variants varying the selection cuts over an extended range, and the stability of our mass measurements when performed on statistically independent subsamples. Table ${ }_{-1}^{-1}$ summarizes the preliminary results on the measurements of masses and widths from the study of $D^{0} \pi^{+}$and $D^{+} \pi^{-}$final states. The $D \pi$ mass spectrum (once subtracted the background, the $D_{2}^{*}$ signal, and the expected feed-downs) shows an excess of events centered around $2420 \mathrm{MeV} / c^{2}$ and about $185 \mathrm{MeV} / c^{2}$ wide. A broad $\left(\sim 100-200 \mathrm{MeV} / c^{2}\right)$ state (the $\left.D_{0}^{*}\right)$ is predicted by HQS at about $2350 \mathrm{MeV} / c^{2}$. The observed excess could be reminiscent of this state, or of a feed-down from another broad state such as the $D_{1}\left(j_{q}=1 / 2\right)$, possibly interfering.

The $D_{s 2}^{*}$ meson has been observed in only one decay mode sofar, $D^{0} K^{+}$. The other allowed decay modes are $D^{+} K_{S}^{0}, D^{* 0} K^{+}$and $D^{*+} K_{S}^{0}$. To search for this state, we considered the decay chains $D_{s 2}^{*} \rightarrow D^{0} K^{+}$, followed by $D^{0} \rightarrow K^{-} \pi^{+}, K^{-} \pi^{+} \pi^{-} \pi^{+}$. Event selection,

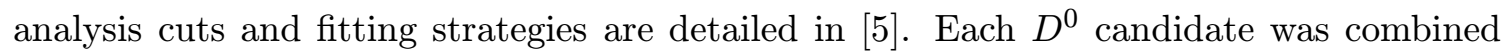
with each $K^{+}$candidate in the same event, which was consistent with coming from the 

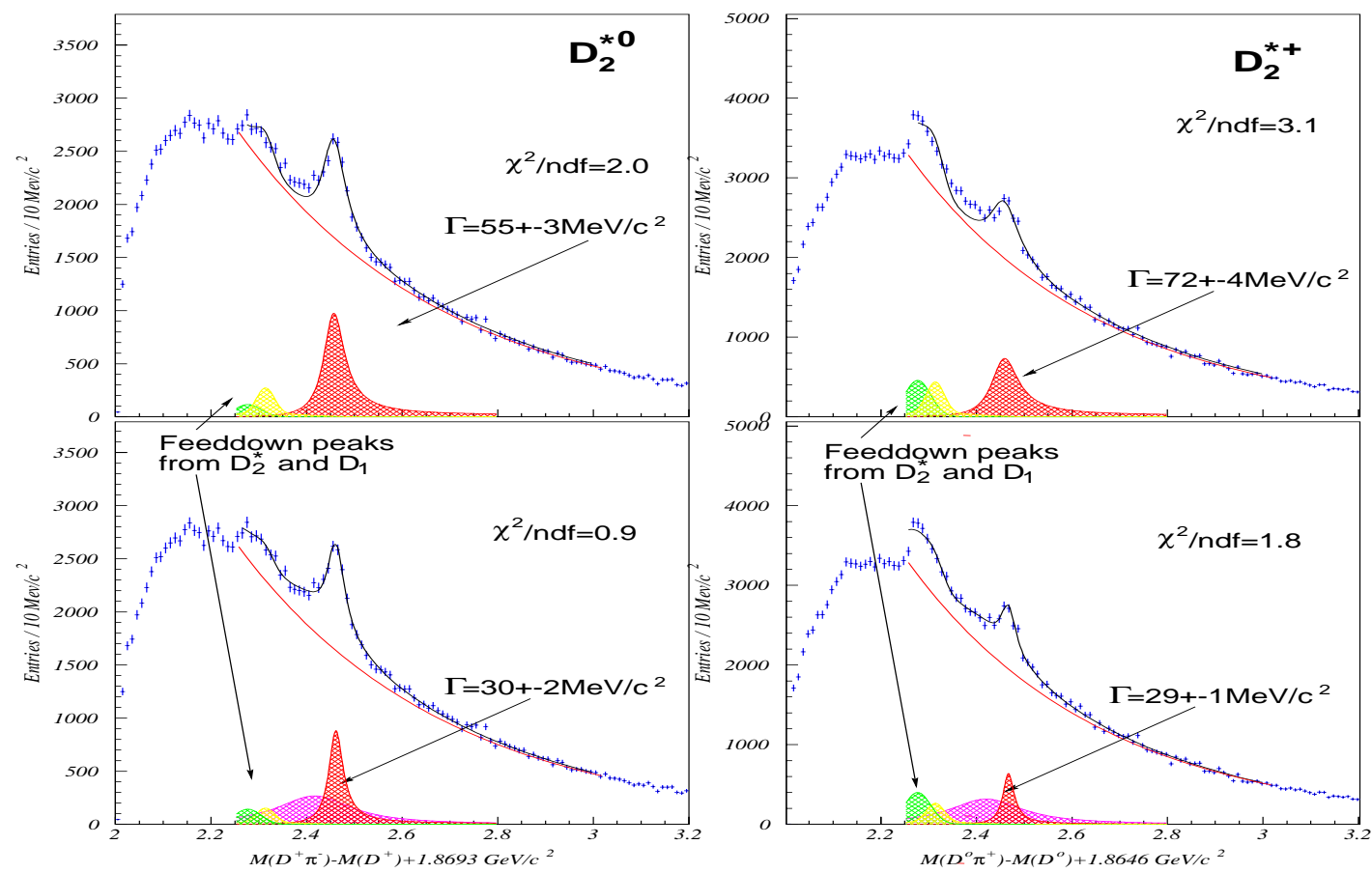

Figure 1: The $D^{+} \pi^{-}\left(D^{0} \pi^{+}\right)$mass spectra is shown on the left (right). The invariant mass variable is defined as $\Delta M_{0} \equiv M\left(D^{+} \pi^{-}\right)-M\left(D^{+}\right)+M_{P D G}\left(D^{+}\right)$and $\Delta M_{+} \equiv M\left(D^{0} \pi^{+}\right)-M\left(D^{0}\right)+$ $M_{P D G}\left(D^{0}\right)$, respectively. In each of the top plots, the mass spectrum was fitted with a relativistic $D$-wave Breit-Wigner function, convoluted with a gaussian resolution function, and the sum of an exponential and two gaussians for the background. For each final state, the bottom histogram shows the fit results when a $S$-wave relativistic Breit-Wigner function is added to the above fit functions.

primary vertex. For each $D^{0} K^{+}$combination the mass difference $\Delta M_{s 0}$ was computed, where $\Delta M_{s 0} \equiv M\left[\left(K^{-} \pi^{+}, K^{-} \pi^{+} \pi^{-} \pi^{+}\right) K^{+}\right]-M\left(K^{-} \pi^{+}, K^{-} \pi^{+} \pi^{-} \pi^{+}\right)$. The distribution of $\Delta M_{s 0}$ for all combinations which pass the analysis cuts is shown as the data points in Fig. The prominent structure near a mass difference of $0.7 \mathrm{GeV} / \mathrm{c}^{2}$ corresponds to the $\bar{D}_{s 2}$, while the narrow structure near a mass difference of $0.525 \mathrm{GeV} / \mathrm{c}^{2}$ arises from a feeddown process, $D_{s 1} \rightarrow D^{* 0} K^{+}$followed by $D^{* 0} \rightarrow D^{0} \pi^{0}$ or $D^{* 0} \rightarrow D^{0} \gamma$. To search for the insofar unobserved decay $D_{s 2} \rightarrow D^{+} K_{S}^{0}$, we used the decay chain $D_{s 2} \rightarrow D^{+} K_{S}^{0}$ followed by $D^{+} \rightarrow K^{-} \pi^{+} \pi^{+}$and $K_{S}^{0} \rightarrow \pi^{+} \pi^{-}$. For each $D^{+} K_{S}^{0}$ combination the mass difference $\Delta M_{s+}$ was computed, where $\Delta M_{s+} \equiv M\left[\left(K^{-} \pi^{+} \pi^{+}\right) K_{S}^{0}\right]-M\left(K^{-} \pi^{+} \pi^{+}\right)$. The distribution of $\Delta M_{s+}$ is shown in Fig. 2 is $\left.\mathrm{b}\right)$. The prominent structure near a mass difference of $0.7 \mathrm{GeV} / \mathrm{c}^{2}$ is the first observation of the decay $D_{s 2} \rightarrow D^{+} K_{S}^{0}$. The feeddown peak due to $D_{s 1} \rightarrow D^{*+} K_{S}^{0}$ followed by $D^{*+} \rightarrow D^{+} \pi^{0}$ or $D^{*+} \rightarrow D^{+} \gamma$ is barely visible near a mass difference of $0.525 \mathrm{GeV} / \mathrm{c}^{2}$.

Finally, we studied the $D_{s 1}$ via the decay chain $D_{s 1} \rightarrow D^{*+} K_{S}^{0}$, followed by $D^{*+} \rightarrow$ $D^{0} \pi^{+}$. For each $D^{*+} K_{S}^{0}$ combination the mass difference $\Delta M_{s+}^{*}$ was computed, in analogy with the other channels. The distribution of $\Delta M_{s+}^{*}$ for all candidates which passed the selection cuts is shown as data points in Fig. 2 

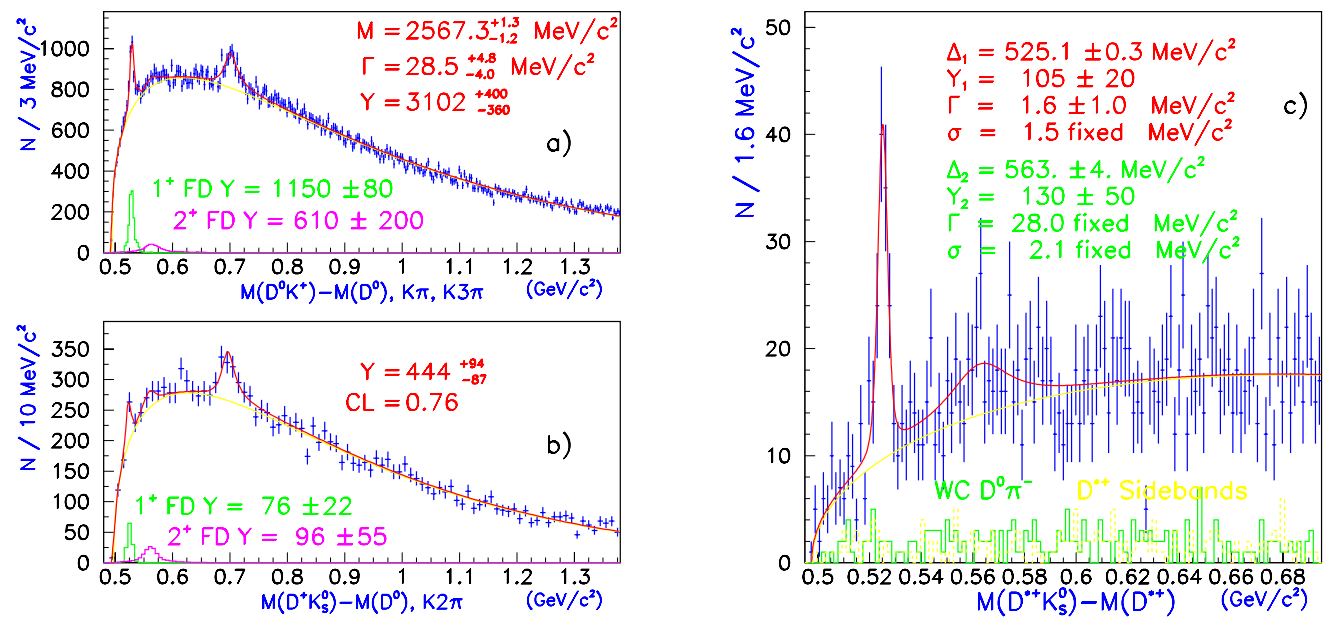

Figure 2: Mass difference distributions for $D^{0} K^{+}, D^{+} K_{S}^{0}$ and $D^{*+} K_{S}^{0}$ combinations. Errors are statistical only.

$0.525 \mathrm{GeV} / \mathrm{c}^{2}$ is the well established $D_{s 1}$. The signal from $D_{s 2}^{*} \rightarrow D^{*+} K_{S}^{0}$ is predicted near $\Delta M_{s+}^{*} \simeq 0.56 \mathrm{GeV} / \mathrm{c}^{2}$ with a $\Gamma \simeq 30 \mathrm{MeV} / \mathrm{c}^{2}$. There is no clear evidence for such a structure in the data but such a structure has been allowed for in the fit. Tab. $\prod_{1}^{\overline{1}}$ gives a summary of the FOCUS mass and width measurements of $\mathrm{L}=1$ charmed-strange mesons.

\section{A narrow dip in a study of diffractive photoproduction.}

The Fermilab experiment 687[i] [i] $]$, FOCUS predecessor, collected data during the 1990/91 fixed-target runs at the Wideband Photon beamline at Fermilab. Although the experiment was focussed on charm physics, a very large sample of diffractively photoproduced lightmeson events was also recorded. We report on a study of the diffractive photoproduction of the $3 \pi^{+} 3 \pi^{-}$final state and the observation of a narrow dip in the mass spectrum at $1.9 \mathrm{GeV} / \mathrm{c}^{2}$. Pions are produced in photon interactions in the Be target. The data acquisition trigger requires a minimum energy deposition in the hadron calorimeters located behind the electromagnetic calorimeters and at least three charged tracks outside the pair region. Details of event selection, analysis cuts and fitting strategies are reported in "6 $6 \overline{1}$. The invariant mass distribution of diffractively produced $3 \pi^{+} 3 \pi^{-}$final states shows a dip structure at about $1.9 \mathrm{GeV} / \mathrm{c}^{2}$. No evidence for structures is shown in the incoherent $\left(P_{T}^{2}>\right.$ $0.040 \mathrm{GeV} / \mathrm{c}^{2}$ subsample (Fig. correcting for efficiency and acceptance, and unfolding the spectrometer mass resolution. The dip structure has been characterized by a two-component fit, adding coherently a relativistic Breit-Wigner resonance to a diffractive continuum contribution. Fit values show consistent evidence for a narrow resonance at $\mathrm{M}_{r}=1.911 \pm 0.004 \pm 0.001 \mathrm{GeV} / \mathrm{c}^{2}$ with a width $\Gamma=29 \pm 11 \pm 4 \mathrm{MeV} / \mathrm{c}^{2}$, where the errors quoted are statistic and systematic, respectively. Such a resonance could be assigned the photon quantum numbers $\left(\mathrm{J}^{\mathrm{PC}}=\right.$ 
$1^{--}$) and $\mathrm{G}=+1, \mathrm{I}=1$ due to the final state multiplicity. There is little understanding of the specific mechanism responsible for this destructive interference.
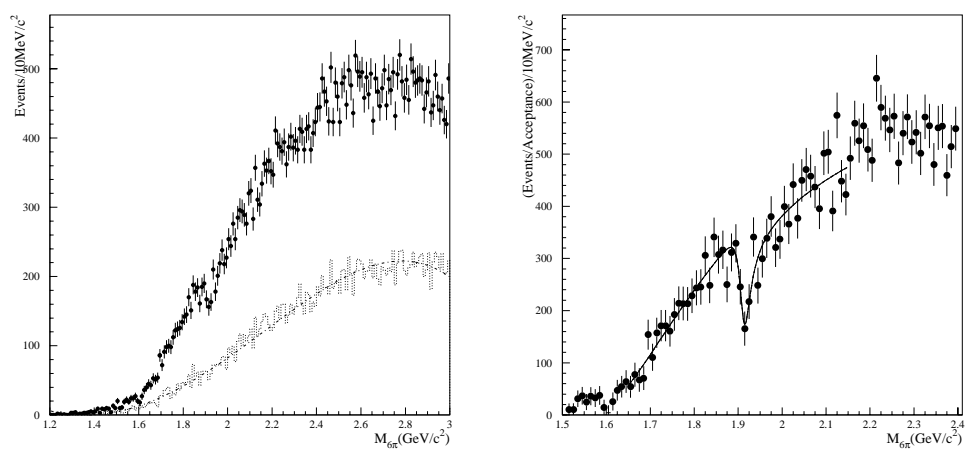

Figure 3: a) Distribution of $3 \pi^{+} 3 \pi^{-}$invariant mass in the $1.2-3.0 \mathrm{GeV} / \mathrm{c}^{2}$ mass range: coherent plus incoherent contribution. Dotted distribution: incoherent contribution. b) Acceptancecorrected distribution of $3 \pi^{+} 3 \pi^{-}$invariant mass for diffractive events. The mass resolution has been unfolded.

\section{Acknowledgments}

We wish to acknowledge the assistance of the staffs of Fermi National Accelerator Laboratory, the INFN of Italy, and the physics departments of the collaborating institutions. This research was supported in part by the U. S. National Science Foundation, the U. S. Department of Energy, the Italian Istituto Nazionale di Fisica Nucleare and Ministero dell'Università e della Ricerca Scientifica e Tecnologica, the Brazilian Conselho Nacional de Desenvolvimento Científico e Tecnológico, CONACyT-México, the Korean Ministry of Education, and the Korean Science and Engineering Foundation.

\section{References}

[1] S. Godfrey and N. Isgur, Phys. Rev. D32, 189 (1985); S. Godfrey and R. Kokoski, Phys. Rev. D43, 1679 (1991); N. Isgur and M.B. Wise, Phys. Rev. Lett. 66, 1130 (1991); E.J. Eichten, C.T. Hill and C. Quigg, Phys. Rev. Lett. 71, 4116 (1993); J. Bartelt and S. Shukla, Annu. Rev. Nucl. Part. Sci 199545 133-61.

[2] F. L. Fabbri, In *Frascati 1999, Hadron spectroscopy* 627-639; S. Bianco, hep-ex/9911034.

[3] P. L. Frabetti et al. (E687 Coll.), NIM A320, 519 (1992), NIM A329, 62 (1993).

[4] S. Sarwar et al. [FOCUS Collaboration], arXiv:hep-ex/0011072.

[5] R.Kutschke, Proc. Frontiers in Contemp. Phys. II - Nashville, TN (USA), 5-10 March, 2001.

[6] P. L. Frabetti et al. (E687 Coll.), Phys. Lett. B 514 (2001) 240 arXiv:hep-ex/0106029. 